\title{
Sinopsis de las especies de Pyracantha (Rosaceae, Maloideae) naturalizadas en la Argentina
}

\author{
Synopsis of Pyracantha (Rosaceae, Maloideae) species naturalized \\ in Argentina
}

Adriel I. Jocou ${ }^{1 *}\left[\right.$ y Ricardo Gandullo ${ }^{1}[0$

1. Departamento de Biología Aplicada. Facultad de Ciencias Agrarias, Universidad Nacional del Comahue. Río Negro, Argentina.

*adrieljocou@gmail.com

Citar este artículo JOCOU, A. I. \& R. GANDULLO. 2019. Sinopsis de las especies de Pyracantha (Rosaceae, Maloideae) naturalizadas en la Argentina. Bol. Soc. Argent. Bot. 54: 599-616.

DOI: http://dx.doi. org/10.31055/1851.2372.v54. n4.24970

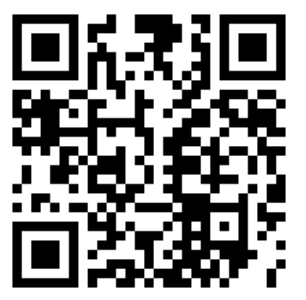

Recibido: 18 Julio 2019

Aceptado: 13 Septiembre 2019 Publicado: 15 Diciembre 2019 Editor: Franco Ezequiel Chiarini (D)

ISSN versión impresa 0373-580X ISSN versión on-line 1851-2372

\section{SUMMARY}

Background and aims: The genus Pyracantha has about ten species, widespread as ornamental, naturalized and considered invasive in different parts of the world. Historically, the identification of taxa has been difficult. Three species were reported as naturalized in Argentina, however, no genus revision has been carried out in the country until now. In order to make new contributions to the Argentinean Flora, given the complexity of the genus and the lack of an integral work in this regard, the objective of this contribution is to present a synopsis of the Pyracantha species naturalized in Argentina.

M\&M: Specimens of each species from different national and international herbaria were studied, and were contrasted with the type specimens. The original descriptions and specific bibliography of each taxon was reviewed.

Results: A summary of the five naturalized species is presented. Distribution maps in Argentina are presented in addition to descriptions, a key, diagrams of the main leaf morphological characters and a series of images that allow the identification of the species. Lectotypes for four names are designated.

Conclusions: Five species are naturalized in Argentina. P. fortuneana and $P$. koidzumii constitute new records for the flora of the country. Up to this moment, $P$. fortuneana represents the species with the most austral distribution and, together with $P$. angustifolia, are the most distributed species in Argentina. P. koidzumii is restricted mainly to the center-east of Argentina. $P$. atalantioides and $P$. coccinea are the less represented species.

\section{KEY WORDS}

Alien, Argentinean Flora, invasive, firethorn, lectotypification, naturalization, Pyracantha.

\section{RESUMEN}

Introducción y objetivos: El género Pyracantha posee alrededor de diez especies, difundidas como ornamentales, naturalizadas y consideradas invasoras en diferentes partes del mundo. Históricamente, la identificación de los taxones ha sido dificultosa. Tres especies fueron reportadas como naturalizadas en la Argentina, sin embargo, no se ha efectuado una revisión del género en el país hasta el momento. Con el fin de realizar nuevos aportes a la Flora Argentina, dadas la complejidad del género y la falta de un trabajo integral al respecto, el objetivo de esta contribución es presentar una sinopsis de las especies de Pyracantha naturalizadas en la Argentina.

M\&M: Se estudiaron ejemplares de cada especie de diversos herbarios nacionales e internacionales, y se contrastaron con los ejemplares tipo. Se revisaron las descripciones originales y bibliografía específica de cada taxón.

Resultados: Se presenta un resumen de las cinco especies naturalizadas. Se proveen mapas de distribución en la Argentina, además de descripciones, una clave, esquemas de los principales caracteres morfológicos foliares y una serie de imágenes que permiten la identificación de las especies. Se designan lectotipos para cuatro nombres.

Conclusiones: Cinco especies se encuentran naturalizadas en la Argentina. $P$. fortuneana y $P$. koidzumii constituyen nuevos registros para la flora del país. Hasta el momento, $P$. fortuneana representa la especie con distribución más austral y, junto a $P$. angustifolia, son las especies más distribuidas en la Argentina. $P$. koidzumii se encuentra restringida principalmente al centro-este del país. $P$. atalantioides y $P$. coccinea son las especies menos representadas.

\section{Palabras clave}

Crataegus, exóticas, Flora Argentina, invasoras, lectotipificación, naturalización, Pyracantha. 


\section{INTRODUCCIÓN}

El género Pyracantha M. Roem. posee alrededor de diez especies, originarias de Europa y Asia (Dimitri, 1972; Robertson et al., 1991; Lance \& Zika, 2014; Csurhes et al., 2016), algunas de ellas naturalizadas en África, Australia y Norteamérica (Lance \& Zika, 2014; Csurhes et al., 2016) y consideradas malezas o invasoras (Csurhes et al., 2016; Randall, 2017). Las especies de este género son difundidas principalmente como ornamentales por su follaje perenne, abundante floración y llamativa fructificación (Montani, 1962; Dimitri, 1972; Aedo et al., 1998; Cheers, 1999).

Históricamente, la identificación de los taxones ha sido dificultosa por su variabilidad, afinidad intraespecífica y la existencia de híbridos y cultivares reportados por Meyer et al. (1994) y Egolf \& Andrick (1995). Esta situación se ha visto reflejada a nivel mundial en la identificación errática de gran cantidad de ejemplares de herbario y en las numerosas publicaciones de nuevas especies de Pyracantha a finales del siglo XIX y principios del siglo XX.

En la Argentina, la naturalización de Pyracantha angustifolia (Franch.) C. K. Schneid. y P. coccinea M. Roem. fue reportada hace más de 20 años (Moschione \& Klimaitis, 1988; Delucchi, 1991), mientras que $P$. atalantioides (Hance) Stapf. fue registrada recientemente (Cantero et al., 2016). En el centro del país Giorgis et al. (2011a) han mencionado la presencia de $P$. aff. atalantioides y $P$. angustifolia y tres años después Giorgis \& Tecco (2014) citaron para la misma zona a $P$. coccinea y $P$. angustifolia.

El relevamiento de la flora de la ribera del río Limay en la provincia de Neuquén, evidenció la naturalización de una nueva especie de Pyracantha en la Argentina. Ante la importancia de comunicar dicho hallazgo, la problemática que presenta la diferenciación de las especies y la falta de un trabajo integral al respecto, el objetivo de esta contribución es presentar una sinopsis de las especies de Pyracantha naturalizadas en Argentina, aportando una clave dicotómica, descripciones, distribución e ilustraciones; con el fin de estabilizar las determinaciones de los ejemplares.

\section{Materiales y Métodos}

Los datos incluidos en este trabajo se obtuvieron de la revisión de numerosos ejemplares de diferentes herbarios de la Argentina: ARC, BA, BAA, BAB, BAL, CTES, CORD, MCNS, MERL, SI, SRFA, LIL, LP (siglas según Thiers, 2019) y de trabajos de campo realizados entre 2018 y 2019.

Para la identificación de las especies se consultó bibliografía específica, protólogos originales y ejemplares tipo. Se estudió material adicional de diversos herbarios internacionales: A, DES, E, FLAS, K, KANU, L, NY, P, PE, PRE, TALL, TROY, USF, UWAL, WAG (siglas según Thiers, 2019) disponibles en diferentes bases de datos on-line.

Bajo las normas del CIN (Turland et al., 2018), se propone la lectotipificación de cuatro nombres: Cotoneasterangustifolius (EPyracantha angustifolia), Pyracantha gibbsii (= Pyracantha atalantioides), Photinia fortuneana (三 Pyracantha fortuneana) y Pyracantha crenulata var. yunnanensis (= Pyracantha fortuneana).

En este trabajo, se considera como especie naturalizada a toda planta exótica que se autoperpetúa durante numerosos ciclos de vida sin intervención humana, sin ser necesariamente invasoras de ecosistemas (Richardson et al., 2000; Pyšek et al., 2004). Se considera planta invasora a toda especie naturalizada que produce descendencia de manera abundante y posee el potencial de extender su área de dispersión a distancias considerables de las plantas progenitoras (Richardson et al., 2000). Se sigue el concepto de maleza de Richardson et al. (2000), como toda planta que crece en sitios donde no se desea y que generalmente tiene efectos económicos o ambientales detectables.

Los ejemplares coleccionados en la Argentina procedentes de poblaciones naturalizadas se citan bajo el título "material estudiado" y se utilizaron para presentar la distribución de cada especie dentro del territorio argentino. Bajo el título "material adicional examinado" se citan ejemplares cultivados en la Argentina y ejemplares representativos del exterior.

Se realizó una breve descripción de cada especie, más detallada en los nuevos registros. Asimismo, se presenta una clave y esquemas de los principales caracteres foliares, para facilitar la identificación de las especies naturalizadas en la Argentina.

\section{Resultados}

A partir de este estudio, se eleva a cinco el total de especies presentes en el país. Pyracantha 


\section{A. I. Jocou y R. Gandullo - Especies de Pyracantha en la Argentina}

fortuneana (Maxim.) H. L. Li se reporta a partir de ejemplares coleccionados en el norte de la Patagonia argentina, depositados en el herbario ARC, y de algunos ejemplares erróneamente identificados de los herbarios CORD y MCNS. Pyracantha koidzumii (Hayata) Rehder, se reporta formalmente a partir de ejemplares de Entre Ríos (CTES, LP), Buenos Aires (LP) y de diversos ejemplares de Córdoba (CORD) con una identificación original errónea.
Se presentan los mapas de distribución geográfica de Pyracantha angustifolia (Fig. 1A), P. atalantioides (Fig. 1B), P. coccinea (Fig. 1C), P. fortuneana (Fig. 1D) y P. koidzumii (Fig. 1E).

Se provee una clave dicotómica y esquemas de los principales tipos de hojas (Figs. 2-3) para la diferenciación de las cinco especies presentes en el país.

Se presenta una serie de fotografías de

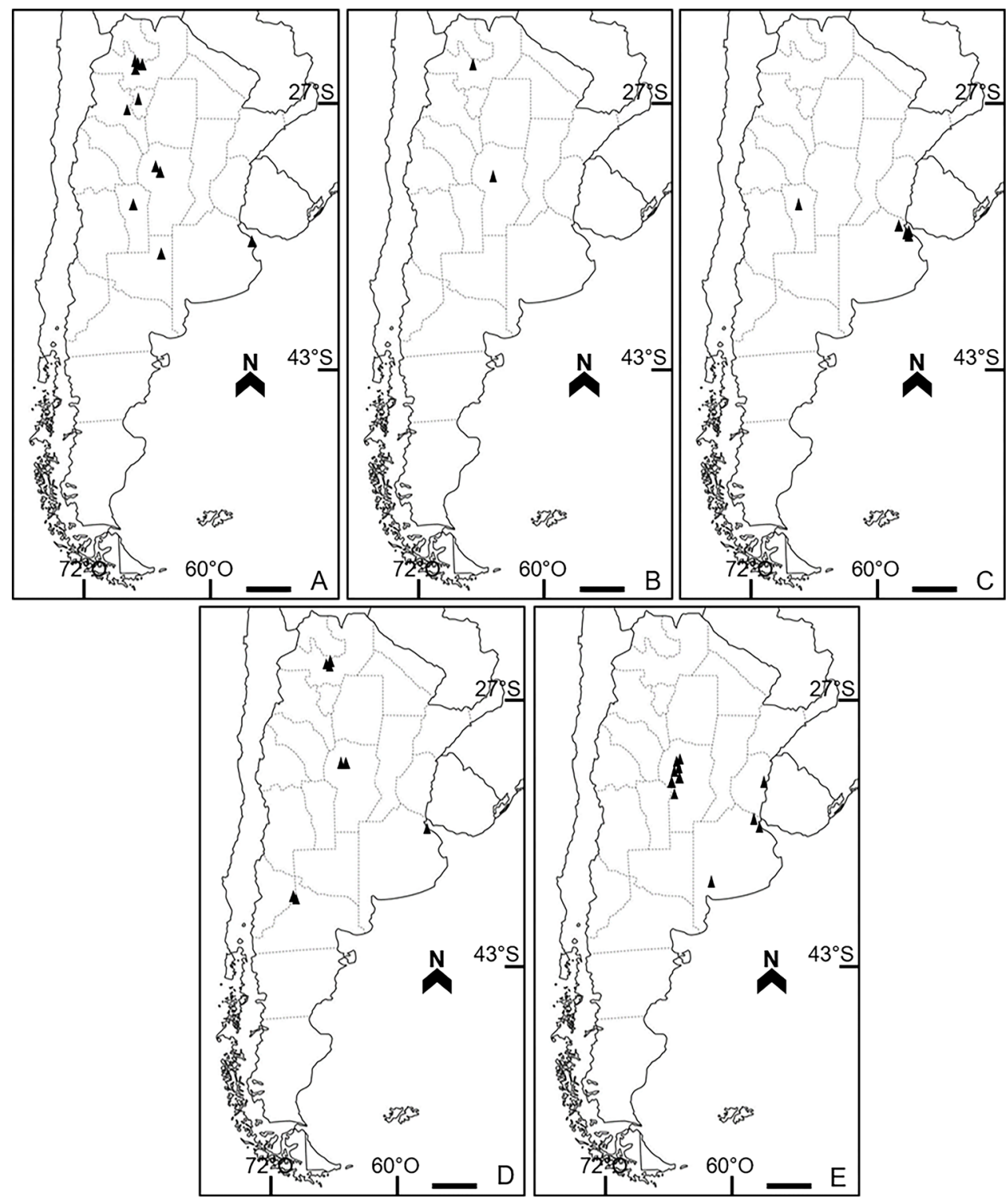

Fig. 1. Zonas de naturalización de las especies de Pyracantha en la Argentina. A: $P$. angustifolia. B: $P$. atalantioides. C: P. coccinea. D: P. fortuneana. E: P. koidzumii. Escala = A-E: $300 \mathrm{~km}$. 
Pyracantha angustifolia (Fig. 4), P. coccinea (Fig. 5), P. fortuneana (Fig. 6) y P. koidzumii (Fig. 7). Las figuras $6 \mathrm{E}-\mathrm{G}$ se obtuvieron con microscopio estereoscópico a partir del material Jocou \& Gandullo 2199 (ARC).

De acuerdo a la revisión bibliográfica, las especies aquí tratadas son muy difundidas en el país por su valor ornamental y se utilizan para cercos vivos de parques y jardines (Montani, 1962; Dimitri, 1972). Se reproducen por semillas, gajos o acodos
(Dimitri, 1972). En la Argentina, son vulgarmente conocidas como "crataegus", "crateus", "crategus" o "espinillo" (Dimitri, 1972; Delucchi, 1991; Peña, 2011).

La información de las etiquetas de ejemplares de herbario y diversos autores (Ridley, 1930; Bass, 1990; Delucchi, 1991; Lepschi, 1993; Peña, 2011; Csurhes et al., 2016; Dellafiore et al., 2016) sugieren que, al consumir los frutos, las aves son el principal factor de dispersión de las especies de Pyracantha.
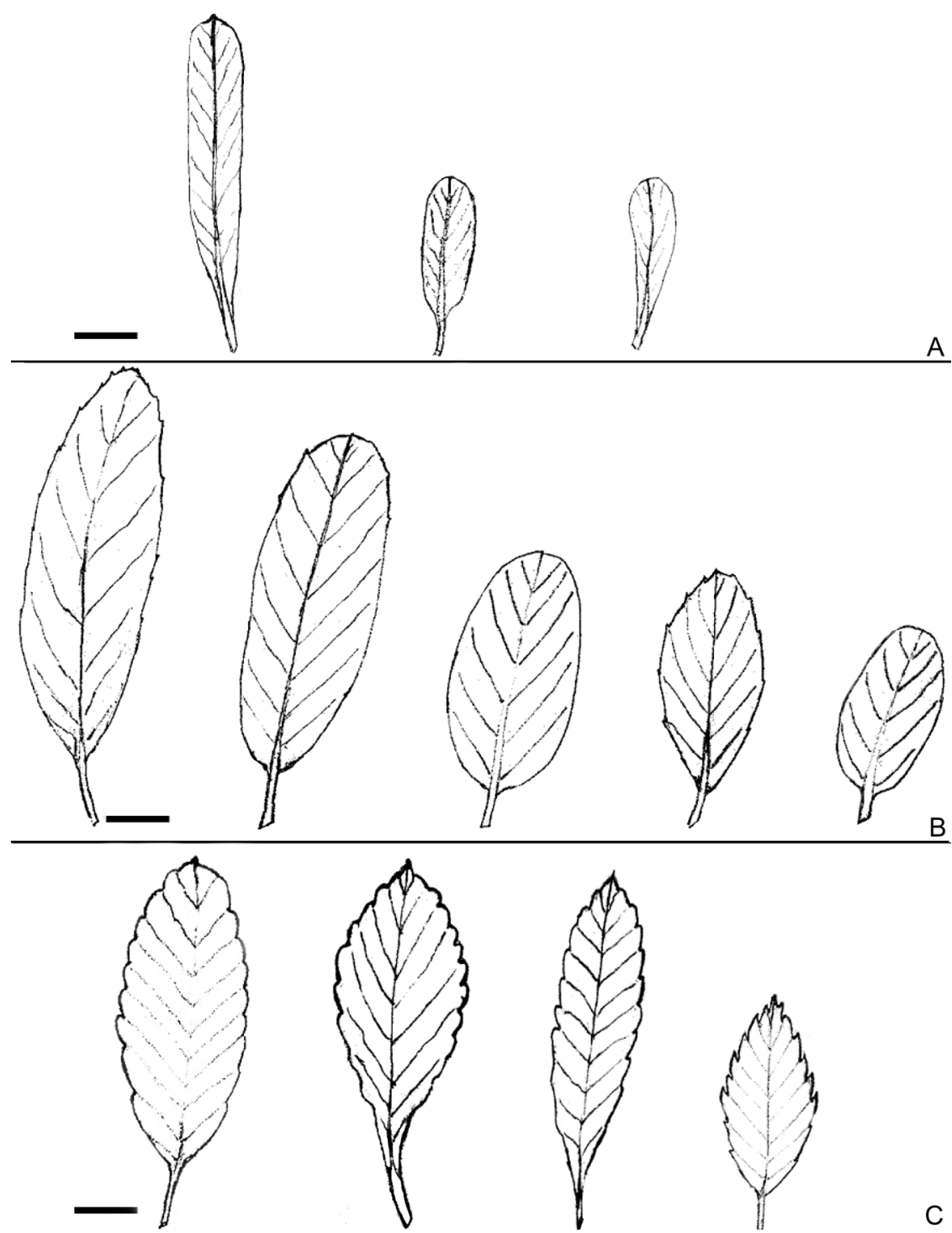

C

Fig. 2. Variación en la morfología foliar de las especies de Pyracantha. A: P. angustifolia. B: P. atalantioides. C: $P$. coccinea. Escalas $=\mathrm{A}-\mathrm{C}: 1 \mathrm{~cm}$. 


\section{A. I. Jocou y R. Gandullo - Especies de Pyracantha en la Argentina}

Peña (2011) reportó el consumo de frutos de $P$. coccinea por diferentes aves: "zorzal chiguanco" (Turdus chiguanco), "cotorra" (Myiopsitta monachus), "benteveo" (Pitangus sulphuratus), "zorzal chalchalero" (Turdus amaurochalinus) y "calandria grande" (Mimus saturninus).

Pyracantha angustifolia (Franch.) C. K. Schneid., Ill. Handb. Laubholzk. 1: 761. 1906. Cotoneaster angustifolius Franch., Pl. Delavay. 221. 1890. TIPO: CHINA. Prov. Yunnan, "Les haies à Li-Kiang", VII-1883, Delavay 61 (Lectotipo aquí designado P 04326863 [foto!]; Isolectotipo P
04326862 [foto!]). Figs. 2A; 4.

Arbusto de 2-5 m alt., con ramas espinescentes. Hojas estrechamente oblongas, 1-6 × 0,4-0,9 $\mathrm{cm}$, tomentosas en la cara abaxial, glabras 0 subglabras en la adaxial, borde entero, ápice obtuso o redondeado, a veces mucronado, base atenuada, pecíolo 1-2 $\mathrm{mm}$ long. Receptáculo pubescente. Sépalos densamente tomentosos, ovados a deltoides, ápice obtuso.

Iconografía. Dimitri (1972: 458, fig. 123C); Montani (1962: fig. 1); Novara (2012: 51, fig.

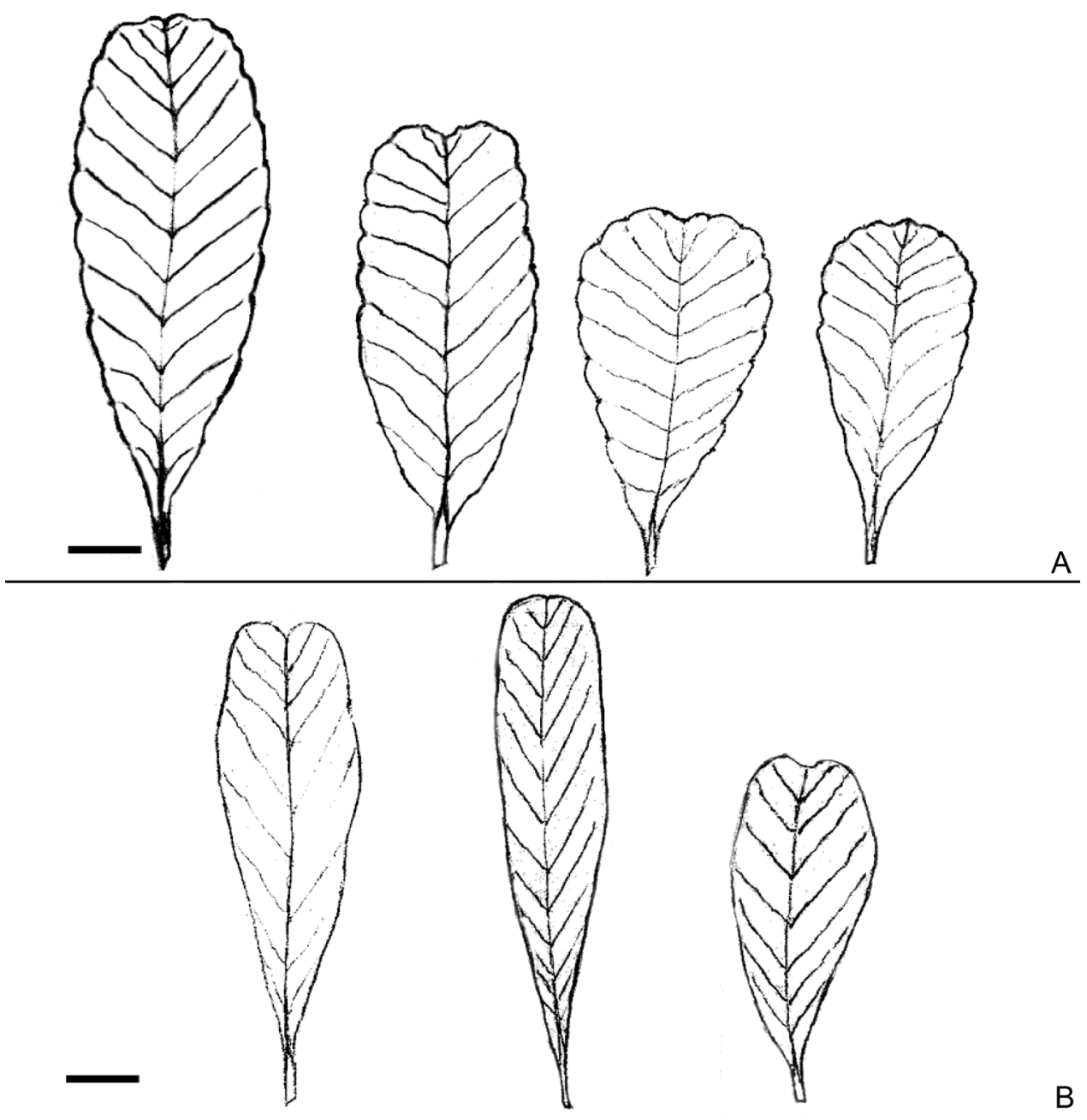

Fig. 3. Variación en la morfología foliar de las especies de Pyracantha. A: P. fortuneana. B: P. koidzumii. Escalas $=\mathrm{A}, \mathrm{B}: 1 \mathrm{~cm}$. 

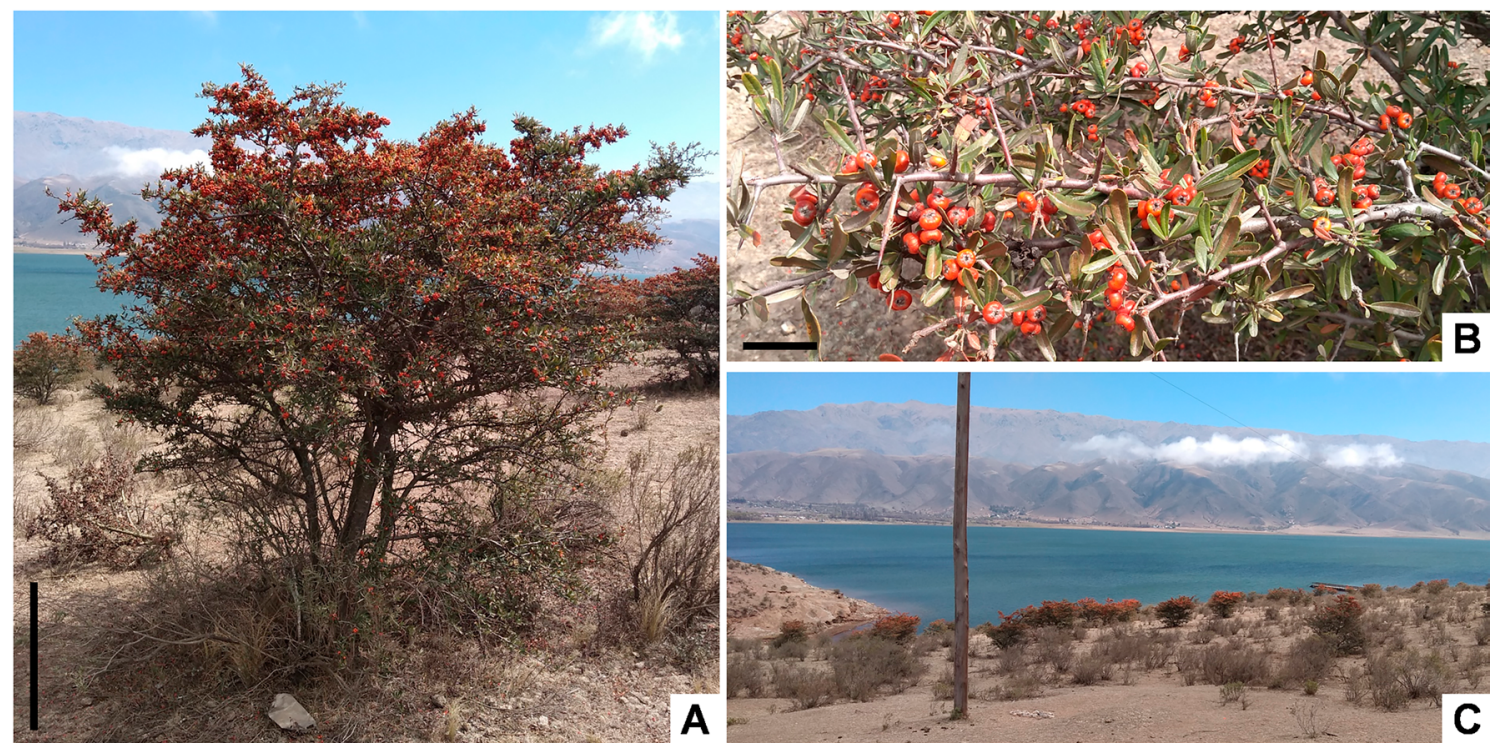

Fig. 4. Pyracantha angustifolia. A: Vista general. B: Rama fructífera, nótese la pubescencia de la cara abaxial de las hojas y de la zona calicinal. C: Vista general de una población. Escalas = A: $40 \mathrm{~cm} ; \mathrm{B}: 5 \mathrm{~cm}$.

13); Prain (1910: fig. 8345, 1-8 y fructificación); Schneider (1906: 762, fig. 430 a-b).

Distribución, hábitat y ecología. Especie nativa de China, naturalizada en los Estados Unidos, Australia, Hawái, Nueva Zelanda y Sudáfrica (Nesom, 2010; Lance \& Zika, 2014). Se trata de la especie más invasora según los reportes y es clasificada por Randall (2017) como maleza de alto riesgo.

En Norteamérica crece en áreas perturbadas, campos abandonados y bordes de caminos (Lance \& Zika, 2014). En China forma matorrales en laderas de montañas (Cuizhi \& Spongberg, 2003).
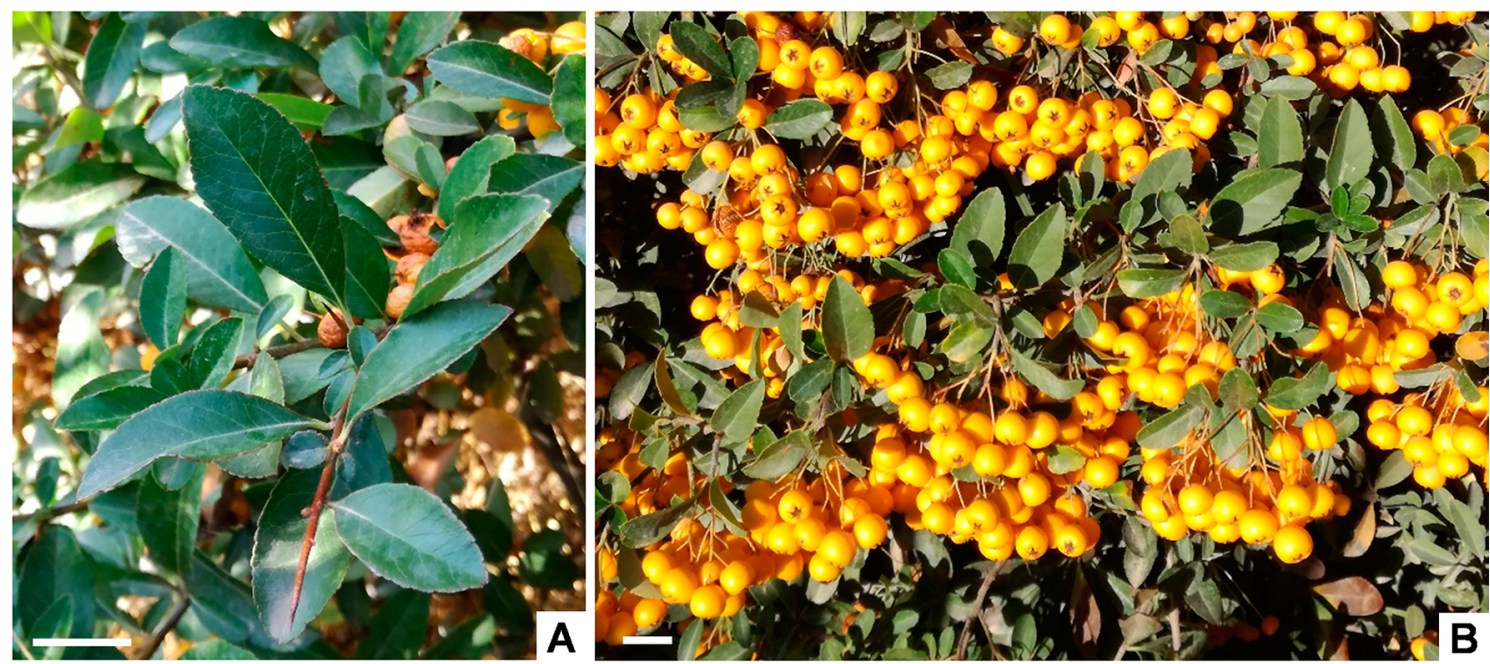

Fig. 5. Pyracantha coccinea. A: Hojas. B: Rama fructifera. Escalas $=A, B: 1,8 \mathrm{~cm}$. 


\section{A. I. Jocou y R. Gandullo - Especies de Pyracantha en la Argentina}
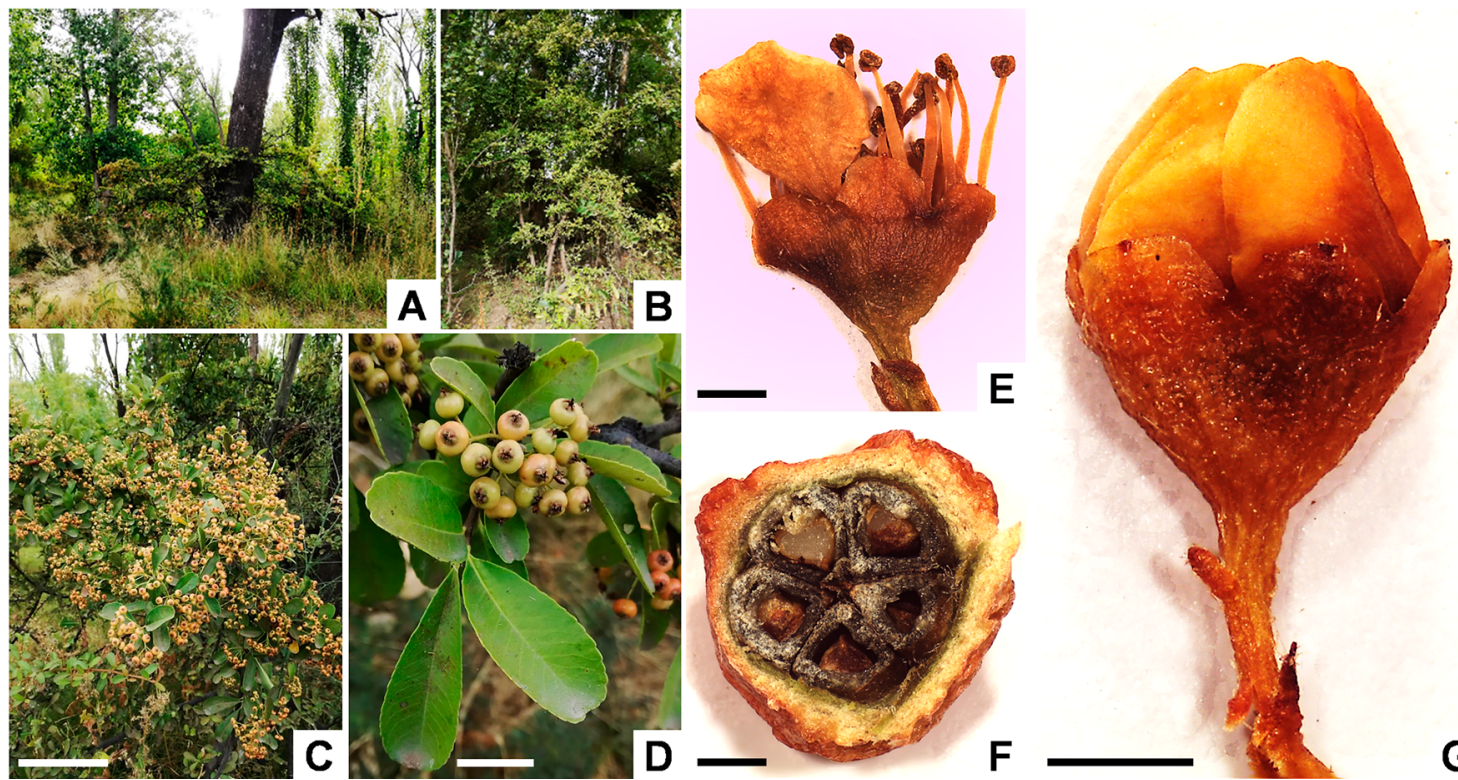

$\mathbf{G}$

Fig. 6. Pyracantha fortuneana. A: Forma de crecimiento lateral en un espacio abierto. B: Forma de crecimiento vertical (hasta de 2,5 m) promovida por el apoyo de otras especies arbóreas exóticas. C: Rama en fructificación. D: Frutos y hojas, nótense los bordes, ápice y base de las mismas. E: Flor, nótese el receptáculo glabro. F: Fruto deshidratado en sección transversal, nótense los 5 pirenos monospermos. G: Botón floral, nótense la forma de los sépalos y el ápice de los mismos. Escalas = C: $10 \mathrm{~cm} ; \mathrm{D}: 2,5 \mathrm{~cm}$; E-G: $1 \mathrm{~mm}$.
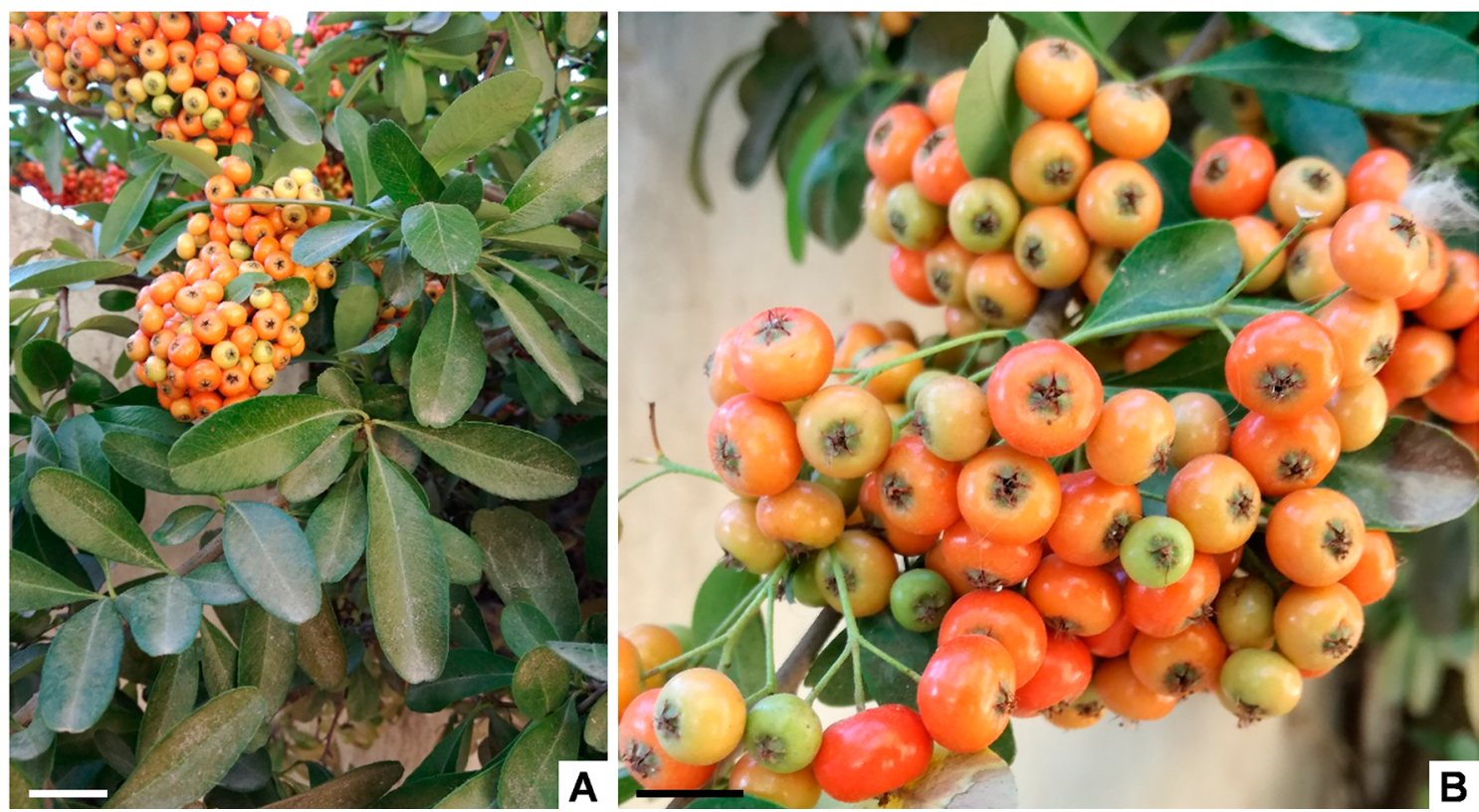

Fig. 7. Pyracantha koidzumii. A: Hojas y fructificación. B: Frutos. Escalas = A: $2,5 \mathrm{~cm} ; \mathrm{B}: 1 \mathrm{~cm}$. 
En Argentina, fue citada para la provincia de Córdoba (Delucchi, 1991; Giorgis \& Tecco, 2014; Vergara Tabares et al., 2016) y Salta (Novara, 2012). Además, según registros de herbario, se encuentra naturalizada en las provincias de Buenos Aires, Catamarca, La Pampa, San Luis y Tucumán. Suele estar asociada a cursos de agua (Delucchi, 1991; Novara, 2012). Se halla muy extendida en matorrales y pastizales del centro del país (Tecco et al., 2006; Giantomasi et al., 2008; Giorgis et al., 2011b; Zeballos et al., 2014). Las observaciones de herbario sugieren que en Salta crece en las Yungas, pastizales serranos húmedos, zonas de transición entre Chaco Serrano y Yungas, quebradas húmedas con selvas en galería y compite con la vegetación nativa.

Observación I. Franchet (1890) ha mencionado en el protólogo de Cotoneaster angustifolius tres materiales, sin designar un holotipo. Durante la revisión de material se hallaron los sintipos en el herbario P. Los datos de las etiquetas de los ejemplares Delavay 3730 (P 04326867) y Delavay 47 (P 04326864) coinciden con los datos del protólogo original. Sin embargo, se ha detectado un error corregible en la cita del material Delavay 61, al mencionar juntos los ejemplares (Delavay, n. 61 et 47) y no especificar que corresponden a un año de colección distinto. Debido a que no se han encontrado duplicados de Delavay 3730 y Delavay 47 , se optó por designar aquí como lectotipo al ejemplar Delavay 61 (P 04326863), con $\mathrm{su}$ respectivo isolectotipo (P 04326862). Dichos ejemplares coinciden en su morfología con la diagnosis original, presentan muy buen estado de conservación y permiten visualizar claramente los caracteres taxonómicos.

Observación II. Numerosos ejemplares que han sido erróneamente identificados como Pyracantha angustifolia corresponden a $P$. koidzumii, sin embargo, la primera se distingue fácilmente por la pubescencia característica en la cara adaxial de las hojas, el receptáculo y el cáliz.

Material estudiado. ARGENTINA. Prov. Buenos Aires: Pdo. Punta Indio, Punta Indio, Sarandí, 12X-2002, Delucchi 2593 (LP). Prov. Catamarca: Dpto. Andalgalá, Inmediaciones del Río Potrero, 12-XII-1995, Hunziker et al. 25530 (CTES). Prov. Córdoba: Dpto. Punilla, cerca de Tanti, 20-
VII-2015, Giorgis 1773 (CORD); camino a Tanti, entrada Los Chorillos, 20-VII-2015, Giorgis 1778 (CORD). Prov. La Pampa: Dpto. Capital, Santa Rosa, 2-II-1996, Steibel \& Troiani 1182 (SRFA). Prov. Salta: Dpto. La Caldera, Los Yacones, 2-XI1989, Juárez 1937 (CTES, MCNS, BAB); Yacones, $500 \mathrm{~m}$ finales del camino antes de la tranquera de la Finca Yacones, 13-XII-1998, Novara et al. 11142 (CTES, MCNS); Yacones, ladera de cerros al W del camino y río, 29-IV-1990, Novara \& Bruno 9824 (MCNS); Finca Castellanos, $2 \mathrm{~km}$ al W del camino que conduce a Yacones, 12-III-1996, Tolaba \& Martínez 676 (MCNS). Dpto. Rosario de Lerma, Río Blanco, $2 \mathrm{~km}$ al $\mathrm{W}$ de Rosario de Lerma, ingresando a Quebrada del Toro, aledaños al Río Blanco y del puente del ramal ferroviario C-14, 11-V-2006, Novara \& Bruno 12676 (MCNS); Quebrada del Toro, frente a Río Blanco, 7-IV-1987, Novara 6494 (MCNS). Dpto. Capital, Ciudad de Salta, XI-1984, Zabala 1322 (MCNS); próximo a la Rotonda de Limache, 8-II-1982, Novara 2339 (MCNS); San Lorenzo, laderas al norte del pueblo y del margen izquierdo del $\mathrm{A}^{\circ}$ San Lorenzo, 1-2 km al NE de la entrada a la Quebrada homónima, 8-I1998, Novara 10963 (MCNS); Finca Castellanos, 12-III-1996, Martínez 255 (MCNS); Parque San Martín, 12-XII-1983, Lozano 88 (MCNS). Prov. San Luis: Dpto. La Capital, Estancia Grande, El Reparito, 29-XI-1986, Del Vitto et al. 1635 (LP). Prov. Tucumán: Dpto. Tafi del Valle, El Mollar, 13IX- 2019, Gandullo GR832 (ARC); Tafí del Valle, a orillas del río, cerca del puente de La Banda, 1-XII2000, Figueroa et al. 1420 (LIL).

Material adicional examinado. ARGENTINA. Prov. Buenos Aires: Pdo. Balcarce, Estación Experimental Regional Agropecuaria, 19-IV-1977, s. c. 260, BAL5791 (BAL). Prov. Jujuy: Dpto. Capital, San Salvador de Jujuy, Jardín de Facultad de Ciencias Agrarias, 8-XI-1985, Ahumada 5227 (MCNS). CHINA. Prov. Fujian: Shenei, 20-VI-1939, Liou 11184 (PE). Prov. Hubei: Enshí, 25-VI-1958, Mingyuan 24447 (PE). Prov. Yunnan: Condado Yongping, Beidou, 15-VII-2009, Shudong \& Kexian 90507 (PE). SUDÁFRICA. Prov. Gauteng: Gran Johannesburgo, Roodepoort, Rooikrans, 2-V-1979, Duggan \& Henderson 55 (PRE). Prov. Free State: Dist. Thabo Mofutsanyane, Bethlehem, Saulspoort Dam, between office and angling sites near wall, 31V-1994, Glen 3615 (BA). 


\section{A. I. Jocou y R. Gandullo - Especies de Pyracantha en la Argentina}

Pyracantha atalantioides (Hance) Stapf., Bot. Mag. 151: sub tab. 9099. 1926. Sportella atalantioides Hance, J. Bot. 15: 207. 1877. TIPO: CHINA. Prov. Cantón, "Secusamnem Lie chau, provinciae Cantonensis, supra pagum Taiwan", X-1876, Nevin 19960 (Holotipo BM 000946993 [foto!]). Fig. 2B.

= Pyracantha gibbsii A. B. Jacks., Gard. Chron. Ser. 3, 60: 309. 1916. TIPO: CHINA. Prov. Hubei, Badong, Patung, "Bush 2'-18' [2 ft to 18 ft] Common. Fls. White", IV-1900, Wilson 349 (Lectotipo aquí designado A 00969287 [foto!]).

= Pyracantha discolor Rehder, J. Arnold Arbor. 1: 261. 1920. TIPO: CHINA. Prov. Hubei, Fang Xian, "side of streams, alt. 1300 m", VI-1907, Wilson 2986 (Holotipo A 00969256 [foto!]).

Arbusto de 1-6 m alt., con ramas espinescentes, ramas jóvenes de color castaño amarillento. Hojas elípticas, oval-elípticas u oblongas, 1,5$5,5 \times 1-2 \mathrm{~cm}$, glabras, borde entero, serrulado u oscuramente serrado, ápice obtuso o redondeado, mucronado, base redondeada a cuneada, pecíolo 2-6 mm long. Receptáculo glabro. Sépalos glabros, pubescentes cuando jóvenes, anchamente elípticos o triangulares, ápice agudo.

Iconografia. Bowles (1918: 443); Fang \& Liao (2006: 462, fig. 682); Gan (2005: 413, fig. 495); Jackson (1916: 309, fig. 133.3; 310, fig. 134).

Distribución, hábitat y ecología. Originaria del sureste y oeste de China, extensamente cultivada en Norteamérica, donde se ha naturalizado (Lance \& Zika, 2014). Esta especie es clasificada como una maleza de bajo riesgo en el listado de Randall (2017).

En Norteamérica y China crece en bordes de caminos, matorrales, laderas, valles, bosques abiertos y terrenos disturbados (Cuizhi \& Spongberg, 2003; Lance \& Zika, 2014). En los Estados Unidos se encuentra naturalizada de forma esporádica en zonas de clima templado (Lance \& Zika, 2014).

En la Argentina se encuentra naturalizada en las provincias de Córdoba y Salta. Las observaciones de herbario sugieren que es muy frecuente sobre la ladera de montaña en La Serranita (Córdoba) y forma parte del piso superior de las Yungas, a los $1700 \mathrm{msnm}$, en el departamento La Caldera (Salta).
Observación I. Al revisar otros sintipos citados por Jackson (1916) y depositados en A, se observó que Wilson ha utilizado un mismo número para colecciones realizadas en diferentes momentos. En este sentido, la colección Wilson 662 posee colecciones de julio y diciembre de 1907 en A y otras sin fecha en el herbario K. Otros ejemplares vistos en A (Wilson 4871a y 2984) pertenecen a otro taxón y no son coincidentes con la diagnosis. Probablemente Wilson asignó ese número a varias colecciones de diferentes especies y podrían no ser sintipos citados por Jackson (1916). El ejemplar Wilson 2986, citado por Jackson, es holotipo de Pyracantha discolor y no puede ser elegido como lectotipo de $P$. gibbsii. El ejemplar Wilson 349 (A 00969287), designado aquí como lectotipo de P. gibbsii, coincide con los datos del material citado, la diagnosis, la ilustración y fotografía del protólogo original. Existe otro ejemplar, Wilson 349 (A 00969288), que no corresponde a la localidad indicada en el protólogo, por lo cual no es un duplicado del anterior y no puede ser considerado como isolectotipo.

Observación II. Numerosos ejemplares de la Argentina identificados originalmente como Pyracantha atalantioides corresponden en realidad a P. koidzumii. Sin embargo, los materiales aquí citados reúnen los caracteres típicos de $P$. atalantioides, con hojas oblongas a elípticas, más anchas hacia la mitad, de bordes enteros a serrulados y ápice obtuso a redondeado, generalmente mucronado. Además, se observó que estos ejemplares presentan variabilidad del borde de la hoja aún dentro del mismo espécimen.

Observación III. En función de los materiales examinados y en concordancia con Nesom (2010), la cara adaxial glaucescente de las hojas, carácter utilizado en la Flora de China (Cuizhi \& Spongberg, 2003), parece ser altamente variable.

Material estudiado. ARGENTINA. Prov. Córdoba: Dpto. Santa María, La Serranita, 12-X1996, Galetto \& Torres 412 (CORD). Prov. Salta: Dpto. La Caldera, Yacones, laderas de cerros al W del camino y río, 29-IV-1990, Novara \& Bruno 9825 (MCNS).

Material adicional examinado. ARGENTINA. Prov. Mendoza: Dpto. Luján de Cuyo, Chacras 
de Coria, 31-III-1964, sin colector 73 (MERL). Dpto. Capital, Parque Gral. San Martín, 1-X-1985, Paladini 444 (MERL). CHINA. Prov. Guandong: Condado Yangshan, Yangshan y alrededores, VII/ IX-1932, Tsui 667 (P). Prov. Guangxi: Condado Luzhai, Mt. Guting, 30-IV-2005, Yang-Ming 101 (P). Prov. Hunan: Condado Changning, 17-VII1935, Fan \& Li 291 (L, P). Condado Dongan, Shunhuangshan, 20-IV-2004, Jin-Kui 452 (NY, P). Condado Xining, 26.4N, 110.8E, 24-IV-1996, Chong-chun 1314 (P, WAG); 2-VIII-1994, Linho 120 (WAG). PAÍSES BAJOS. Prov. Holanda Septentrional, Amsterdam, sin fecha, Westendorp s.n. (WAG); "Sortimentstuin Proefstation", 9-IX1961, van de Laar 952 (WAG).

Pyracantha coccinea M. Roem., Fam. Nat. Syn. Monogr. 3: 219. 1847, nom. cons. TIPO: AUSTRIA. "In Comitatu Tyroliensiadrivum in sabulo", Herb. Burser XXIV: 8 (Lectotipo, UPS BOT: V-175776 [foto!], designado por Muñoz Garmendia \& Aedo, Taxon 47: 171. 1998). Figs. 2C; 5.

Arbusto de 1-6 m alt., con ramas espinescentes. Hojas oblanceoladas, ovadas, sub-rómbicas o elípticas, 1,5-4 × 1-2 cm, glabras, cara adaxial verde oscura, lustrosa, la abaxial más clara, borde serrado o crenado, ápice acuminado o agudo, si obtuso, entonces mucronado, base \pm redondeada o cuneiforme, pecíolo $2-5 \mathrm{~mm}$ long. Receptáculo piloso en la cara externa. Sépalos pilosos, triangulares.

Iconografia. Montani (1962: figs. 2-3); Garsault (1767: fig. 385); Lance \& Zika (2014); Schneider (1906: 762, fig. 430 e-i); Serviss (2009: 320, fig. 1C); Von Kerner (1794: fig. 623).

Distribución, hábitat y ecología. Originaria del sur de Europa y oeste de Asia (Montani, 1962; Aedo et al., 1998; Nesom, 2010). Naturalizada en los Estados Unidos (Delucchi, 1991; Nesom, 2010; Lance \& Zika, 2014), Inglaterra, Sudáfrica, Australia y Japón (Nesom, 2010; Lance \& Zika, 2014). Especie considerada como maleza de riesgo medio según Randall (2017).

En la Península Ibérica forma matorrales de sustitución de encinares, robledales y hayedos, en suelos con buen drenaje y condiciones de buena iluminación (Aedo et al., 1998). En los
Estados Unidos se encuentra en bosques mixtos de coníferas, terrenos perturbados, campos, matorrales y bordes de caminos (Lance \& Zika, 2014).

En la Argentina crece espontáneamente en jardines del noreste de la provincia de Buenos Aires e invade terrenos baldíos de forma esporádica (Delucchi, 1991). Según los registros de herbario, se comporta como invasora en la Reserva Otamendi (provincia de Buenos Aires) y se encuentra naturalizada en la provincia de San Luis.

Observación. Se ha observado un ejemplar de Pyracantha coccinea que corresponde a una selección (Boom 36346, L), con características foliares diferentes a las típicas de la especie, con ápice obtuso, mucronado y borde crenado o serrado-crenado. En este sentido, el ejemplar de Brasil (Hoehne, 4004, CTES) coincide con las características de la selección mencionada, pero se encuentra identificado bajo el nombre de P. crenulata (D. Don) M. Roem. Sin embargo, $P$. crenulata es un taxón pobremente definido $\mathrm{y}$ con identificaciones muy erráticas, pero con características diferentes a las de la selección de $P$. coccinea. De esta manera, es recomendable mantener como $P$. coccinea a los siguientes ejemplares que coinciden con las características de la selección: Albrecht 43 (SI), Baenitz s.n. (SI), Del Vitto 3588 (LP) y Lozano 90 (MCNS).

Material estudiado. ARGENTINA. Prov. Buenos Aires: Pdo. La Plata, Gonnet, calle Bordenave y 15 bis, 14-X-1990, Delucchi 378a (BAA). Pdo. Campana, Reserva Otamendi, sendero Laguna Grande, 26-III-2003, Delucchi 2698 (LP). Prov. San Luis: Dpto. Pringles, Estancia grande, El Reparito, XI-1987, Del Vitto \& Petenatti 3872 (LP).

Material adicional examinado. ARGENTINA. Ciudad Autónoma de Buenos Aires: Jardín Botánico Municipal, 8-X-2002, Hurrell \& Bazzano 4840 (BAB); Facultad de Agronomía, Parque, 11VI-1975, Menini 15416 (BAA). Prov. Buenos Aires: Pdo. La Plata, La Plata, Jardín Zoológico, IV-1940, Cabrera 9778 (SI). Pdo. Morón, Castelar, 24-III-1945, Albrecht 43 (SI). Prov. Salta: Dpto. Capital, Ciudad de Salta, Parque San Martín, 13XII-1983, Lozano 90 (MCNS). Prov. San Luis: Dpto. La Capital, El Chorrillo, V-1989, Del Vitto 


\section{A. I. Jocou y R. Gandullo - Especies de Pyracantha en la Argentina}

3588 (LP). BRASIL. Estado São Paulo: Municipio São Paulo, Pacaembu, 12-XII-1959, Hoehne 4004 (CTES). ESTADOS UNIDOS. Estado Georgia: Condado Forsyth, 1942, s.col., LIL388165 (LIL). Estado Kansas: Condado Douglas, 17-IV-2006, Freeman \& Morse 21461 (KANU); N side of Lawrence, Burcham Park, 4-V-2008, Morse s.n. (KANU). Estado New Jersey: Condado Middlesex, Princeton Nuerseries, 17-XI-1942, E.J.P. s.n. (LIL); 8-VI-1942, E.J.P. s.n. (LIL). Estado Utah: Condado Utah, Utah Valley, Provo, Brigham, Young University campus, 27-V-1986, Holmgren 11080 (NY). ESTONIA. Condado Harju, Tallin, 4-VIII-1970, Paivel s.n. (TALL); 19-VII-1984, Thliom? s.n. (TALL); "Flora Istriaca: Abbazia; in Parkanlagen", 19-V-1902, Baenitz s.n. (SI). PAÍSES BAJOS. Prov. Holanda Septentrional, Aalsmeer, kwekerij Terra Nova (Keessen), 14-XI1958, Boom 36346 (L). TURQUÍA. Prov. Hatay, Dörtyol, 22-IX-1965, Yaltirik 4135 (E). Prov. Istanbul, Belgrade forest, near Burunsuz, 26-VIII1960, Yaltirik s.n., E00408178 (E). Prov. Samsun, Kirazlik, 1966, Tobey 1962 (E). Prov. Sinop, Ayancik, 5-VIII-1965, Arikan 3936 (E).

Pyracantha fortuneana (Maxim.) H. L. Li, J. Arnold Arbor. 25(4): 420. 1944. Photinia fortuneana Maxim., Bull. Acad. Imp. Sci. SaintPétersbourg 19(2): 179. 1873. TIPO: CHINA. "China boreali", 1845, Fortune A69. (Lectotipo aquí designado P 02143286 [foto!], Isolectotipos $\mathrm{K} 000758311$ [foto!], K 000758310 [foto!], MO 2392369 [foto!], P 02143287 [foto!], P 02143288 [foto!], PE 00004610 [foto!]). Figs. 3A; 6.

= Pyracantha crenatoserrata (Hance) Rehder, J. Arnold Arbor. 12(1): 72. 1931. Photinia crenatoserrata Hance, J. Bot. 18: 261. 1880. TIPO: CHINA. Prov. Hubei, Yichang, "juxta oppidum Ichangvere", 1879, Watters 20988. (Holotipo BM 000602129 [foto!]).

= Pyracantha crenulata (D. Don) M. Roem. var. yunnanensis M. Vilm. ex Mottet, Rev. Hort. (Paris) 85(9): 204. 1913. Pyracantha gibbsii A. B. Jacks. var. yunnanensis (M. Vilm. ex Mottet) Osborn, Garden (London, 1871-1927) 83: 138. 1919. Pyracantha yunnanensis (M. Vilm. ex Mottet) Chitt., Gard. Chron. ser. 3 70: 325. 1921. TIPO: ilustración en el trabajo original (Lectotipo aquí designado, pl. sin número, pp. 204, en Mottet (1913)).
Arbusto hasta de $3 \mathrm{~m}$ alt., con ramas laterales cortas espinescentes, ramas jóvenes densamente pubescentes, con pelos anaranjados, las adultas glabras. Hojas obovadas a espatuladas, más anchas en la mitad superior, glabras, 1,5-5,5(-7) $\times 0,5$ $2,5(-3) \mathrm{cm}$, cara adaxial verde oscura, la abaxial más clara, las jóvenes laxamente pubescentes en ambas caras, borde crenado o serrado-crenado, ocasionalmente entero en la porción proximal, ápice obtuso o emarginado, raro mucronado, base cuneada o atenuada, pecíolo $2-5 \mathrm{~mm}$ long. Inflorescencia en corimbo compuesto laxo, 3-6 cm diám.; pedúnculo glabro. Flores ca. $10 \mathrm{~mm}$ diám.; pedicelos glabros o subglabros de 1-6 mm long. Receptáculo campanulado, glabro. Sépalos de 1-1,5 $\times$ 1,2-1,5 mm, persistentes, triangulares, glabros, margen entero, ápice obtuso. Pétalos de 3-4 × 2-3 $\mathrm{mm}$, suborbiculares a ovoides, margen entero, a veces sinuado, ápice redondeado. Estambres ca. 20, filamentos de 2-4 mm long. Ovarios densamente pubescentes apicalmente, biovulados; estilos 5 , casi tan largos como los estambres. Pedicelos fructíferos de 5-10 mm long. Fruto de 4-8 mm diám., rojo anaranjado a rojo, subgloboso, comprimido; pirenos 5 , monospermos a la madurez.

Iconografia. Kun-tsun (1992: 145, fig. 25 [1-4]); Montani (1962: fig. 4); Mottet (1913: 204, pl. sin número); Yintang (2007: 314, fig. 336).

Distribución, hábitat y ecología. Especie nativa del centro y oeste de China (Cuizhi \& Spongberg, 2003; Lance \& Zika, 2014), introducida en los Estados Unidos, Europa, Hawái, Nueva Zelanda y Australia (Nesom, 2010; Lance \& Zika, 2014). Es considerada como maleza de bajo riesgo (Randall, 2017).

En Norteamérica y China es frecuente en ambientes disturbados, bordes de caminos y rutas, acantilados, periferias de bosques, bosques abiertos, áreas ribereñas y matorrales (Cuizhi \& Spongberg, 2003; Lance \& Zika, 2014).

En la Argentina se encuentra naturalizada en las provincias de Buenos Aires, Córdoba, Neuquén, Río Negro, Salta y en la Ciudad Autónoma de Buenos Aires. Se encuentran poblaciones naturalizadas en las orillas del Río Limay (provincias de Neuquén y Río Negro), en bordes de caminos y de lagunas naturales cercanas al mismo río. En dicho ambiente, cohabita principalmente con especies exóticas herbáceas, arbustivas, arbóreas y trepadoras: Asparagus officinalis 
L. var. altilis L., Cotoneaster glaucophyllus Franch., Fraxinus pennsylvanica Marshall ssp. pennsylvanica, Lonicera japonica Thunb. var. japonica, Morus alba L., Populus alba L., P. nigra L., Rosa canina L., Rubus ulmifolius Schott., Salix babylonica L., S. fragilis L., Tamarix ramosissima Ledeb. y Vitis vinifera $\mathrm{L}$. En base a los registros de herbario, en el centro del país crece en las zonas de las sierras de Córdoba, en el departamento Colón, a orillas de arroyos. En la provincia de Salta, crece como parte del piso superior de Yungas en el departamento La Caldera y, en el departamento Capital, como parte de las Yungas y transición con el Chaco Serrano.

Observación I. En el protólogo de Photinia fortuneana, el autor no indicó el holotipo entre los numerosos ejemplares de la colección Fortune A69 referenciada, por lo que todos ellos deben ser considerados sintipos. En este sentido, en el herbario $\mathrm{P}$ se han etiquetado erróneamente como "Type" al ejemplar P02143286 e "Isotype" a los ejemplares P02143287 y P02143288. En consecuencia, es necesario efectuar la lectotipificación, designándose al ejemplar P02143286 por presentar un estado óptimo de conservación y visibilizar los principales caracteres taxonómicos. Si bien la grafía original de las etiquetas de los sintipos consiste únicamente en la expresión "A69", se observó que las etiquetas colocadas adicionalmente (no originales) difieren en la localidad y fecha indicadas en el protólogo. Estos errores son ajenos al autor y corregibles, por lo que los ejemplares deberían referenciarse a los datos del protólogo: año 1845, "China boreali”.

Observación II. En el protólogo de Pyracantha crenulata var. yunnanensis, no se cita explícitamente el material examinado por los autores al momento de realizar la descripción. Mottet (1913) sólo indica que para la descripción se utilizó el mismo material que sirvió de base para la acuarela y la fotografía (“que nous avons relevées sur les échantillons qui ont fourni les éléments de la photographie et de la planche [...]"), sin mencionar el herbario en el cual fue depositado. La revisión de diversos herbarios no permitió comprobar la existencia de dicho material. Además, frases del trabajo de Mottet (1913) como "On jugera, d'ailleurs, de la valeur décorative de cette magnifique variété nouvelle à l'examen de la planche coloriée [...]", "L'arbuste, que nous avons eu le plaisir d'admirer dans sa propriété privée [...]" indicarían que ciertas observaciones se han realizado sobre la acuarela y un ejemplar vivo. Cabe aclarar que el ejemplar depositado en K (K000758631) no puede considerarse como material original, como sugieren las etiquetas ("5012 is Vilmorin's number for P. crenulata v. yunnanensis", "5012 is the no quoted Chittenden by Mottet for P. crenulata v. yunnanensis Vilm"), debido a que proviene de plantas vivas de los jardines del Kew (Renata Borosova, curadora K, com. pers.). Posiblemente esta confusión se haya originado en un error de interpretación, cuando Mottet (1913) indicó que Vilmorin ingresó semillas e introdujo en cultivo la variedad en 1906 bajo el número 5012 (" $\mathrm{La}$ magnifique variété yunnanensis que M. Maurice L. de Vilmorin a eu la bonne fortune de traouver dans ses semis de plantes asiatiques.”, “[...] introduit en culture par les soins de M. Maurice L. de Vilmorin, en 1906, sous le numéro 5012 [...]"). Por los motivos antes mencionados, se efectúa la lectotipificación, designándose la pintura de Mottet (1913: 204, pl. sin número), ya que hasta el momento es el único material original disponible.

Observación III. Los ejemplares de la Argentina estudiados coinciden morfológicamente con el holotipo de Pyracantha crenatoserrata.

Observación IV. Al estudiar el material, se confirmó que algunos ejemplares pertenecientes a Pyracantha fortuneana han sido erróneamente determinados como P. coccinea (Boffa B-5, SI; Novara \& Bruno 9823, Tolaba \& Novara 614, MCNS), P. atalantioides (Hunziker 25612, 25611 y 25605, CORD) o P. angustifolia (Chambi \& Jaime 13279, MCNS). Las hojas obovadas a espatuladas, con borde crenados o serrado-crenados son caracteres típicos de $P$. fortuneana.

Material estudiado. ARGENTINA. Ciudad Autónoma de Buenos Aires, Reserva Costanera Sur, 28-I-1995, Burgueño 204 (LP). Prov. Córdoba: Dpto. Colón, Sierra Chica, camino a La Cumbre, desde Ascochinga, poco después de La Cuesta y del Arroyo Tiumayu (km 46), 31-I-1997, Hunziker 25612 (CORD); camino a La Cumbre, km 46, desde Ascochinga, poco antes de La Cuesta y del Arroyo Tiumayu, 31-I-1997, Hunziker 25611 (CORD); camino de Ascochinga a La Cumbre, poco antes del acceso a la Estancia San Miguel, 31-I-1997, Hunziker 25605 (CORD). Prov. Neuquén: Dpto. Confluencia, 


\section{A. I. Jocou y R. Gandullo - Especies de Pyracantha en la Argentina}

Ciudad de Neuquén, balneario Valentina Sur, 17-XII2018, Jocou \& Gandullo 2199 (ARC); 05-III-2019, Jocou \& Gandullo 2198 (ARC). Prov. Río Negro: Dpto. Gral. Roca, Cipolletti, zona barrio Las Perlas, 05-III-2019, Jocou \& Gandullo 2197 (ARC). Prov. Salta: Dpto. Capital, Quebrada de San Lorenzo, sobre el río San Lorenzo, en parada de colectivo y frente al Castillo, 04-V-2010, Chambi \& Jaime 13279 (MCNS). Dpto. La Caldera, Finca Castellanos, 1-1,5 $\mathrm{km}$ al W del camino Yacones, 14-X-1995, Tolaba \& Novara 614 (MCNS); Yacones, laderas de cerros al W del camino y río, 29-IV-1990, Novara \& Bruno 9823 (MCNS).

Material adicional examinado. ARGENTINA. Prov. Buenos Aires: Pdo. La Plata, La Plata, Facultad de Agronomía, 6-XI-1946, Boffa B-5 (SI). Pdo. Vicente López, Olivos, VI-1947, Dimitri \& Rial Alberti 71551 (BAB). AUSTRALIA. Autralian Capital Territory, Canberra, S.E. base of Black Mountain, 21-XI-1964, McKee 11793 (P); CSIRO Black Mountain site, 25-XI-1998, Craven et al. 10060 (P). CHINA. Prov. Guangxi, Loh Hoh Tsuen, Ling Yün Hsien, 22-V-1933, Steward \& Cheo 475 (P); 14-IV-1933, Steward \& Cheo 209 (P). Prov. Guizhou, Miao Wang, Kiangkou Hsien, 28-IX-1931, Steward et al. 568 (P); Lian Feng Yah, Tsunyi Hsien, 17-VII-1931, Steward et al. 8 (P). Prov. Hunan: Condado Sangzhi, Liangshuikou, 30-V-2010, Zhang et al. 201005300009 (BAB). Prov. Sichuan, Hsientien-tze, in nemore, 23-V-1930, Hummel 3380 (P); Nanchuan Hsien, 1-VI-1928, Fang 1356 (P). ESTADOS UNIDOS. Estado Alabama, Hwy. 263 ca. $31 / 2$ miles south of the Lowndes County line, 10-VIII-2008, Diamond 19742 (TROY, UWAL); junction of US-431 and Blue Mountain Rd, Anniston, 7-XI-2009, Spaulding 13217 (TROY, UWAL). Estado California: Condado Contra Costa, 18-X1942, Bracelin 1730 (LIL). Estado Montana, 1 air mi. NNW of Livingston, Along Hopkins Street just S of Livingston Park, 25-IX-2007, Keener 3871 (TROY, UWAL). Estado Washington, Fremont, Seattle, north bank of Lake Washington Ship Canal, near North Canal Street, 20-X-2007, Zika 23640 (NY).

Pyracantha koidzumii (Hayata) Rehder, J. Arnold Arbor. 1(4): 261. 1920. Cotoneaster koidzumii Hayata, J. Coll. Sci. Imp. Univ. Tokyo 30(1): 101. 1911. TIPO: "Hab. Pinan, Oct. 1896 (A.26)" (Holotipo, no hallado). Figs. 3B; 7.
= Cotoneaster formosanus Hayata, J. Coll. Sci. Imp. Univ. Tokyo 30(1): 101. 1911. TIPO: TAIWÁN. Condado Taitung, "Hab. Tabari, Taito, Taiwan", I-1906, Nakahara 741. (Holotipo PE 00535933 [foto!]).

= Cotoneaster taitoensis Hayata, J. Coll. Sci. Imp. Univ. Tokyo 30(1): 102. 1911. Pyracantha koidzumii var. taitoensis (Hayata) Masam., Annual Rep. Taihoku Bot. Gard. 2: 124. 1932. TIPO: TAIWÁN. Condado Taitung, "Hab. Taito", 1899, Miyake (Holotipo, no hallado).

Arbusto hasta de 3-4 m alt., con ramas laterales cortas espinescentes, ramas jóvenes densamente villosas, con pelos marrones-rojizos, las adultas glabras. Hojas oblanceoladas, estrechamente elípticas o estrechamente obovadas, más anchas entre la mitad y parte distal del limbo, $(1,5-) 2-4(-5,5) \times(0,6-) 1-1,5(-$ 2) $\mathrm{cm}$, glabras, cara adaxial verde oscura, la abaxial verde clara, las jóvenes con pubescencia castaña, borde entero, a veces algo sinuado, raro inconspicuamente serrado o crenado, ápice emarginado o truncado, raro redondeado, base cuneada o atenuada, pecíolo 2-7 mm long. Inflorescencia en corimbo compuesto con abundantes flores, $3-5 \mathrm{~cm}$ diám.; pedúnculo laxamente pubescente. Flores de 6-8 mm diám.; pedicelos con pubescencia laxa castaña de 3-8 mm long. Receptáculo campanulado, pubescente en la porción proximal. Sépalos de 1-1,3 mm long., persistentes, triangulares, glabros, con pubescencia castaña o castaño-amarillenta cuando jóvenes, margen entero, ápice agudo. Pétalos de 3-4 × 3-4 $\mathrm{mm}$, anchamente elípticos a suborbiculares, ápice levemente emarginado. Estambres ca. 20, filamentos de 2-3 mm long. Ovarios pubescentes; estilos 5, casi tan largos como los estambres. Pedicelos fructiferos de 4-14 mm long. Fruto de 5-9 mm diám., rojo anaranjado, subgloboso, comprimido; pirenos 5 .

Iconografia. Ho (1977: 87, fig. 75); Liu (1960: 450, fig. 384); Serviss (2009: 320, fig. 1A).

Distribución, hábitat y ecología. Especie nativa de Taiwán, naturalizada en Hawái, Australia y los Estados Unidos (Nesom, 2010; Lance \& Zika, 2014). Considerada maleza de bajo riesgo según Randall (2017).

En Norteamérica y China crece en bosques disturbados, acantilados de zonas costeras, áreas ribereñas, bordes de caminos, áreas rocosas de valles 
y matorrales (Cuizhi \& Spongber, 2003; Lance \& Zika, 2014).

Según los registros de herbario, en la Argentina crece en Entre Ríos, en el Parque Nacional El Palmar, donde es muy invasora y forma densas matas. En la provincia de Buenos Aires, crece en talares degradados de la Reserva Otamendi, donde es muy frecuente, y en el Parque Provincial Tornquist, en la base del cerro Ventana. Los registros de herbario indican que es muy difundida en la provincia de Córdoba, en ocasiones sobre laderas serranas, al borde de caminos.

Observación. Las hojas con márgenes enteros a subenteros y el ápice emarginado son característicos de Pyracantha koidzumii. Los ejemplares Hurrell \& Bazzano 4839 (BAB) y Delucchi 292 y 381 (BAA), originalmente identificado como $P$. coccinea, y numerosos ejemplares de CORD y LP, identificados como $P$. atalantioides corresponden a $P$. koidzumii.

Material estudiado. ARGENTINA. Prov. Buenos Aires: Pdo. Campana, Reserva Otamendi, sendero Laguna Grande, 26-III-2003, Delucchi 2684 (LP). Pdo. La Plata, Gonnet, 26-III-1989, Delucchi 292 (BAA); calle Bordenave y 15 bis, 14-X-1990, Delucchi 381 (BAA). Pdo. Tornquist, Parque Provincial E. Tornquist, 15-VII-2001, Long 2128 (LP). Pdo. Tres de Febrero, El Palomar, 4-X-2002, Hurrell \& Bazzano 4839 (BAB). Prov. Córdoba: Dpto. Calamuchita, cerca de Los Reartes, 16VIII-2015, Giorgis 1784 (CORD); Giorgis 1783 (CORD). Dpto. Colón, Sierra Chica, camino de Ascochinga a La Cumbre, unos $10 \mathrm{~km}$ al oeste de Ascochinga, después de Tres Cascadas, 31-I-1997, Hunziker 25607 (CORD); camino a La Cumbre, desde Ascochinga (en km 45/46), La Cuesta, cerca de Arroyo Tiumayu, 31-I-1997, Hunziker 25609 (CORD); camino a La Cumbre, desde Ascochinga, poco después de La Cuesta y del Arroyo Tiumayu (km 46), 31-I-1997, Hunziker 25613 (CORD). Dpto. Punilla, Dique San Jerónimo, 31-V-2015, Cantero 7125 (CORD); camino a Tanti, 20-VII-2015, Giorgis 1774 (CORD); El Durazno, desde Tanti a Cerro Blanco y Los Gigantes, 20-VII-2015, Giorgis 1780 (CORD). Dpto. Río Cuarto, Alpa Corral, 19-V-2015, Cantero 7096 (CORD). Dpto. San Alberto, cercanía a Los Hornillos, 23-VIII-2015, Giorgis, 1787 (CORD). Dpto. Santa María, La Serranita, 20-II-1997, Galetto \& Torres 683 (CORD). Prov. Entre Ríos: Dpto.
Colón, Parque Nacional El Palmar, 15-XII-2014, Ferrucci et al. 3314 (CTES); sector de espinal atrás de la intendencia, 2-XI-2008, Delucchi 3333 (LP).

Material adicional examinado. ARGENTINA. Prov. La Pampa: Dpto. Chical Co, La Humada, 05II-2009, Muiño 298 (SRFA). Prov. Río Negro: Dpto. Gral. Roca, General Fernández Oro, 02-IV-2019, Jocou 2200 (ARC). Prov. Tucumán: Dpto. Tafi del Valle, El Mollar, Plaza Central, 2-XII-2014, Muruaga et al. 1802 (LIL). ESTADOS UNIDOS. Estado California: Condado Contra Costa, 7-IX-1941, Bracelin 1634 (LIL); 25-X-1942, Bracelin 2350 (LIL). Condado Santa Cruz, ca. 2 miles south of Hank-Yank Spring, Sycamore Canyon, Pajarito Mountains, 03-XI-1984, Rheinlander et al. 3369 (DES). Condado Los Ángeles, Big Rock Creek, just above the confluence of Holcomb Canyon Creek, 11-VI-1990, Ross 2988 (NY). Estado Florida: Condado Alachua, Kanapaha Botanical Gardens, The Rock Garden, 28-III-2003, Barry Davis 676 (FLAS); Gainesville, University of Florida, 17XI-2004, Hopkins 56 (FLAS). Condado Dade, the Metrozoo on SW side of Miami, 02-X-1983, Nee 28636 (NY). Condado Hillsborough, 2517 Marquette Avenue, Auburn, Highlands, Tampa, 10VI-1970, Burch \& Chevalier 6372 (USF). Condado Hernando, in coastal brushland, Hernando Beach, 19-IX-1981, Correll \& Correll 52541 (USF); on the south side of the park road from parking lot to canoe/kayak concession, 13-VII-2009, Van Hoek 0033 (USF). Condado Walton, at the S edge of De Funiak Springs, along N side of Bruce Ave, just W of $2^{\text {nd }}$ St., 25-XI-1980, Wilhelm 8091 (USF). Condado Okaloosa, ca. $2 \mathrm{mi}$. S of Crestview, 18-V-1983, Wilhelm 11495 (USF). Condado Suwanee, near Suwanee Springs, 04-VII1984, Whetstone 14335 (USF). Condado Pasco, 5 mi. SW of N end of westernmost fence surrounding Woodland Elementary School, 26-XI-1986, Wise 249 (USF). Condado Volusia, Pierson Quad, 21-IV-2010, Slaughter 17718 (USF). Estado Carolina del Sur: Condado Richland, Columbia, N side of Duncan St. at jct with Kilbourne Rd., 26-XII-1994, Nelson 16226 (NY). Estado Texas: Condado Travis, City of Austin, Lady Bird Lake (formerly Town Lake) formed by the Colorado River, NE shore of lake, 03-VIII-2012, Atha 12265 (NY). MÉXICO. Estado Nuevo León, Monterrey, I.T.E.S. campus, 8-VI-2010, Whitlatch s.n. (LIL). REPÚBLICA DOMINICANA. Prov. La 


\section{A. I. Jocou y R. Gandullo - Especies de Pyracantha en la Argentina}

Vega: en la zona "turística" de casa veraniegas de "Racket Club", aprox. 9 km al N de Jarabacoa, en la carretera a La Vega, 26-III-1990, Zanoni \& Jiménez 44262 (NY). TAIWÁN. Condado Taitung: Litao, 04-X-1997, Wu et al. 1535 (PE). Condado Hualien: Yuli, 06-IV-1985, Lu 15697 (PE).

\section{Consideraciones finales}

Si bien suele mencionarse que la morfología foliar de Pyracantha es muy variable (aún dentro de un mismo ejemplar), al revisar una importante cantidad de material se pudo observar, que existe un patrón determinado en cada especie. Al estudiar un ejemplar se suele evidenciar cierta variabilidad en la morfología foliar, pero en la gran mayoría de los casos se destaca una morfología predominante que permite la identificación de la especie con la clave aquí aportada. En ocasiones, la mayor variabilidad se da en el tamaño de las hojas, sin afectar al resto de los caracteres. El principal inconveniente se presenta en plantas no naturalizadas, principalmente cultivadas, donde la presencia de híbridos y cultivares dificulta la clara delimitación de taxones. No se han observado ejemplares naturalizados que presenten características poco definidas o intermedias entre una $\mathrm{u}$ otra especie, y que, consecuentemente, no puedan ser identificados con la clave propuesta.
Para garantizar una mayor facilidad en el proceso de identificación de las especies, se recomienda estudiar las hojas con mayor desarrollo. En este sentido, se aconseja que, para futuras colecciones de Pyracantha, se incluyan ramas con hojas en su máximo desarrollo y representativas de la planta de la cual fueran colectadas.

Sin duda, son necesarios estudios taxonómicos y moleculares que permitan establecer límites más exactos entre taxones y comprender más en profundidad al género Pyracantha.

\section{Conclusiones}

Con base en los registros actuales es posible concluir que cinco especies de Pyracantha se encuentran naturalizadas en la Argentina, de las cuales $P$. fortuneana y $P$. koidzumii constituyen nuevos reportes para la flora del país.

Hasta el momento, Pyracantha fortuneana es la especie con distribución más austral y junto a $P$. angustifolia son las más distribuidas en la Argentina. P. koidzumii se encuentra restringida principalmente al centro-este del país. $P$. atalantioides y $P$. coccinea son las especies menos representadas.

\section{Clave para diferenciar las especies de Pyracantha naturalizadas en la Argentina}

1. Hojas adultas menores a $1 \mathrm{~cm}$ de ancho, tomentosas en la cara adaxial, receptáculo y sépalos densamente pubescentes Pyracantha angustifolia

$1 '$. Hojas adultas generalmente mayores a $1 \mathrm{~cm}$ de ancho, nunca tomentosas en la cara adaxial, receptáculo y sépalos nunca densamente pubescentes

2

2. Hojas, en su mayoría de bordes lisos, raro serrulados u oscuramente serrados, nunca marcadamente

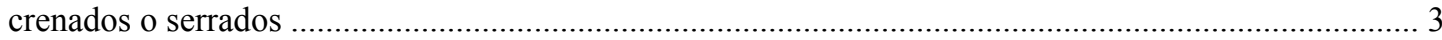

$2^{\prime}$. Hojas en su mayoría de bordes marcadamente crenados, serrados o crenado-serrados 4

3. Hojas elípticas, oblongas u oblongo-ovadas ensanchadas en la mitad de su longitud, de ápice entero, excepcionalmente emarginado, frecuentemente mucronado, bordes lisos, serrulados u oscuramente serrados dentro de la misma planta

Pyracantha atalantioides

3'. Hojas oblanceoladas o estrechamente obovadas, ensanchadas por encima de la mitad de su longitud, de ápice frecuentemente emarginado, raro mucronado, bordes lisos, a veces espaciada e inconspicuamente serrados en el tercio superior Pyracantha koidzumii

4. Hojas ensanchadas en la mitad, ovadas a elípticas, bordes marcadamente serrados, si crenados entonces ápice mucronado, normalmente agudo, acuminado o bien obtuso Pyracantha coccinea

4'. Hojas ensanchadas distalmente, obovadas a espatuladas, bordes crenados o crenado-serrados, nunca notablemente serrados, ápice obtuso a trunco, a veces emarginado, mútico Pyracantha fortuneana 


\section{Contribución de los autores}

Ambos autores participaron conjuntamente y en partes iguales tanto en la colecta de datos y material vegetal como en la redacción del manuscrito. Los mapas, dibujos y fotografías en Figs. 1-3 y 5-7 de A. Jocou y fotografías Fig. 4 de R. Gandullo.

\section{Agradecimientos}

A los revisores anónimos por sus valiosas sugerencias que permitieron enriquecer el manuscrito y a Carlos Minué por la lectura crítica del mismo. A S. Donadío por sus sugerencias nomenclaturales. A A. Bartoli (BAA), A. López (BAL), A. Suarez (SI), A. P. Wiemer (CORD), A. Voda, M. Monsalvo y R. Fortunato (BAB), A. Prina (SRFA), C. Alvarenga (BA), G. Pieszko (CTES), L. Iharlegui (LP), L. Bonjour (MERL), N. Muruaga (LIL) y O. Martínez (MCNS) por proveer las imágenes de los ejemplares de Pyracantha. A M. Hjertson (UPS) por facilitar las imágenes del lectotipo de $P$. coccinea. A A. Brach (A) por proveer la imagen del holotipo de $P$. discolor y de los materiales originales de $P$. gibbsii. A C. Schollaardt (L) por proveer la imagen del ejemplar de P. coccinea. A M. Jeanson (P) por su gran ayuda para localizar los materiales originales de $P$. angustifolia. A V. Andro (P) por su sugerencias y aclaraciones sobre el material original de P. fortuneana. A R. Borosova (K) por la información brindada sobre la procedencia del ejemplar de P. yunnanensis. A G. Benito (Jardín Botánico Carlos Thays) por proveer la bibliografía solicitada para este manuscrito. El presente trabajo se financió con fondos de la Secretaría de Ciencia y Técnica de la Universidad Nacional del Comahue.

\section{Biblografía}

AEDO, C., F. MUÑOZ GARMENDIA \& C. NAVARRO. 1998. Pyracantha. En: Muñoz Garmendia, F. \& C. Navarro (eds.), Flora Ibérica: Plantas vasculares de la Peninsula Ibérica e Islas Baleares, vol. 6, pp. 391394. CSIC, Madrid.

BASS, D. A. 1990. Dispersal of an introduced shrub (Crataegus monogyna) by the Brush-tailed Possum (Trichosurus vulpecula). Austral. J. Ecol. 15: 227-229.

https://doi.org/10.1111/j.1442-9993.1990.tb01531.x
BOWLES, E. A. 1918. Pyracantha gibbsii. Garden (London) 82: 443. Disponible en: https://www. biodiversitylibrary.org/item/82699

CANTERO, J. J., G. E. BARBOZA, F. E. CHIARINI, R. DEANNA, L. ARIZA ESPINAR, M. A. GIORGIS, C. O. NÚÑEZ \& G. BERNARDELLO. 2016. Novedades para la flora de la Argentina. Bol. Soc. Argent. Bot. 51: 183-207. https://doi.org/10.31055/1851.2372.v51.n1.14488

CHEERS, G. 1999. Botanica, the illustrated A-Z of over 10,000 garden plants and how to cultivate them. 3ra ed. Könemann, Milsons Point.

CSURHES, S., J. WEBER \& Y. ZHOU. 2016. Invasive plant risk assessment: Firethorn. Pyracantha species. Queensland Government, Queensland.

CUIZHI, G. \& S. A. SPONGBERG. 2003. Pyracantha. In: WU, Z. Y., P. H. RAVEN \& D. Y. HONG (eds.) Flora of China, vol. 9, pp. 108-111. Science Press, Beijing, and Missouri Botanical Garden Press, St. Louis. Disponible en: eFloras.org

DELLAFIORE, C. M., M. J. ROSA \& V. SCILINGO. 2016. ¿Afectan las aves la germinación del arbusto Pyracantha atalantioides (Rosaceae)?. Cuad. Inv. UNED 7: 295-299. https://doi.org/10.22458/urj.v7i2.1156

DELUCCHI, G. 1991. Especies adventicias y naturalizadas nuevas o críticas para Argentina. Bol. Soc. Argent. Bot. 27: 179-181.

DIMITRI, M. J. 1972. Pyracantha. En: DIMITRI, M. J. (dir.), Enciclopedia Argentina de Agricultura y Jardinería, $2^{\text {da }}$ ed., vol. 1, pp. 456-457. ACME, Buenos Aires.

EGOLF, D. R. \& A. O. ANDRICK. 1995. A checklist of Pyracantha cultivars. U.S. National Arboretum Contribution, $\mathrm{n}^{\circ}$ 8. U.S. Govt. Printing Office, Washington. https://doi.org/10.5962/bhl. title. 58687

FANG, Z. X. \& C. L. LIAO. 2006. Medicinal flora of Enshi. Hubei Science and Technology Press, Wuhan. Disponible en: https://www. biodiversitylibrary.org/page/36802745

FRANCHET, A. 1890. Plantae Delavayanae. Paul Klincksieck Libraire, Paris. https://doi.org/10.5962/bhl.title.10529

GAN, Q. 2005. Flora Zhuxiensis. Hubei Science and Technology Press, Wuhan. Disponible en: https:// www.biodiversitylibrary.org/page/36694002

GARSAUlT, F. A. P. DE 1767. Traité des plantes et animaux, tomo 3. P. F. Didot, París. https://doi.org/10.5962/bhl.title.69123 


\section{A. I. Jocou y R. Gandullo - Especies de Pyracantha en la Argentina}

GIANTOMASI, A., P. A. TECCO, G. FUNES, D. E. GURVICH \& M. CABIDO. 2008. Canopy effects of the invasive shrub Pyracantha angustifolia on seed bank composition, richness and density in a montane shrubland (Córdoba, Argentina). Austral Ecol. 33: 6877. https://doi.org/10.1111/j.1442-9993.2007.01791.x

GIORGIS, M. A. \& P. A. TECCO. 2014. Árboles y arbustos invasores de la provincia de Córdoba (Argentina): una contribución a la sistematización de bases de datos globales. Bol. Soc. Argent. Bot. 49: 581-603.

GIORGIS, M. A., A. M. CINGOLANI, F. CHIARINI, J. CHIAPELLA, G. BARBOZA, L. ARIZA ESPINAR, R. MORERO, D. E. GURVICH, P. A. TECCO, R. SUBILS \& M. CABIDO. 2011a. Composición florística del Bosque Chaqueño Serrano en la provincia de Córdoba, Argentina. Kurtziana 36: 9-43.

GIORGIS, M. A., P. A. TECCO, A. M. CINGOLANI, D. RENISON, P. MARCORA \& V. PAIARO. 2011b. Factors associated with woody alien species distribution in a newly invaded mountain system of central Argentina. Biol. Invasions 13: 1423-1434. https://doi.org/10.1007/s10530-010-9900-y

HO, F. C. 1977. Tropical plants of Taiwan in color. Hengchun Tropical Botanical Garden, Pingtung. Disponible en: https://www.biodiversitylibrary.org/ item/113220

JACKSON, A. B. 1916. Two new pyracanthas from China. Gard. Chron. Ser. 3, 60: 309-310.

KUN-TSUN, F. (ed.). 1992. Flora Loess-Plateaus Sinicae. Tomus 2. Instituto Botanico Borealioccidentali. China Forestry Publishing House, China. Disponible en: https://www.biodiversitylibrary.org/ page/36662186

LANCE, R. W. \& P. F. ZIKA. 2014. Pyracantha. In: Flora of North America Editorial Committee (eds.), Flora of North America, vol. 9, pp. 469-472. Oxford University Press, New York. Disponible en: eFloras.org.

LEPSCHI, B. J. 1993. Food of some birds in Eastern New South Wales: Additions to Barker \&Vestjens. EmuAustral Ornithology 93: 195-199. https://doi.org/10.1071/MU9930195

LIU, T. S. 1960. Illustrations of native and introduced ligneous plants of Taiwan: DicksoniaceaeUlmaceae, vol. 1. College of Agriculture - National Taiwan University, Taipei. Disponible en: https:// www.biodiversitylibrary.org/item/156124

MEYER, F. G., P. M. MAZZEO \& D. H. VOSS. 1994. A Catalog of Cultivated Woody Plants of the Southeastern United States. U.S. National
Arboretum Contribution, $n^{\circ}$ 7. U.S. Govt. Printing Office, Washington. https://doi.org/10.5962/bhl.title.58811

MONTANI, R. G. 1962. Las especies del género Pyracantha (Rosaceae) cultivadas en la Argentina. Revista Inst. Munic. Bot. 2: 31-41.

MOSCHIONE, F. N. \& J. KLIMAITIS. 1988. Flora de Punta Lara. Cartilla sistemática de los vegetales superiores de la Reserva de Selva Marginal de Punta Lara y alrededores (Provincia de Buenos Aires). Grupo de Observadores Ribereños de Aves.

MOTTET, S. 1913. Un nouveau buissonardent: le Pyracantha crenulata et sa variété yunnanensis. Rev. Hort. (Paris) 9: 204-205.

NESOM, G. L. 2010. Pyracantha (Rosaceae) naturalized in Texas and the southeastern United State. Phytoneuron 2010: 1-6.

NOVARA, L. 2012. Flora del Valle de Lerma: Rosaceae. Aportes Bot. Salta, Ser. Fl., edición digital, vol. 2, 11: 1-53.

PEÑA, M. R. DE LA 2011. Observaciones de campo en la alimentación de las Aves. Biológica 13: 1-88.

PRAIN, D. 1910. Pyracantha angustifolia. Bot. Mag. [sic]. ser. 4, 71: tab. 8345. Disponible en: https:// www.biodiversitylibrary.org/item/14262

PYŠEK, P., D. M. RICHARDSON, M. REJMÁNEK, G. L. WEBSTER, M. WILLIAMSON \& J. KIRSCHNER. 2004. Alien plants in checklists and floras: towards better communication between taxonomists and ecologists. Taxon 53: 131-143. https://doi.org/10.2307/4135498

RANDALL, R. P. 2017. A Global Compendium of Weeds. 3rd Edition. Perth, Western Australia.

RICHARDSON, D. M., P. PYŠEK, M. REJMÁNEK, M. G. BARBOUR, F. D. PANETTA \& C. J. WEST. 2000. Naturalization and invasion of alien plants: concepts and definitions. Diversity \& Distrib. 6: 93-107. https://doi.org/10.1046/j.1472-4642.2000.00083.x

RIDLEY, H. N. 1930. The dispersal of plants throughout the world. L. Reeve \& Co., Ashford.

ROBERTSON, K. R., J. B. PHIPPS, J. R. ROHRER \& P. G. SMITH. 1991. A synopsis of genera in Maloideae (Rosaceae). Syst. Bot. 16: 376-394. https://doi.org/10.2307/2419287

SCHNEIDER, C. K. 1906. Illustriertes Handbuch der Laubholzkunde. Verlag von Gustav Fischer, Jena. https://doi.org/10.5962/bhl.title.194

SERVISS, B. E. 2009. Pyracantha koidzumii (Rosaceae) new to the Arkansas Flora. J. Bot. Res. Inst. Texas 3: 319-321. 
TECCO, P. A., D. E. GURVICH, S. DÍAZ, N. PÉREZHARGUINDEGUY \& M. CABIDO. 2006. Positive interaction between invasive plants: The influence of Pyracantha angustifolia on the recruitment of native and exotic woody species. Austral Ecol. 31: 293-300. https://doi.org/10.1111/j.1442-9993.2006.01557.x

THIERS, B. 2019. Index Herbariorum: A global directory of public herbaria and associated staff. New York Botanical Garden's Virtual Herbarium. Disponible en: http://sweetgum.nybg.org/science/ih [consulta: 15 junio 2019].

TURLAND, N. J., J. H. WIERSEMA, F. R. BARRIE, W. GREUTER, D. L. HAWKSWORTH, P. S. HERENDEEN, S. KNAPP, W. H. KUSBER, D. Z. LI, K. MARHOLD, T. W. MAY, J. MCNEILL, A. M. MONRO, J. PRADO, M. J. PRICE \& G. F. SMITH (eds.). 2018. International Code of Nomenclature for algae, fungi, and plants (Shenzhen Code) adopted by the Nineteenth International Botanical Congress Shenzhen, China, July 2017. Regnum Vegetabile 159.
Koeltz Botanical Books, Glashütten. https://doi.org/10.12705/Code.2018

VERGARA TABARES, D. L., J. BADINI \& S. I. PELUC. 2016. Fruiting phenology as a "triggering attribute" of invasion process: Do invasive species take advantage of seed dispersal service provided by native birds? Biol. Invasions 18: 677-687. https://doi.org/10.1007/s10530-015-1039-4

VON KERNER, J. S. 1794. Abbildung aller ökonomischen Pflanzen. Cotta, Stuttgart. Disponible en: http://mdz-nbn-resolving.de/ urn:nbn:de:bvb:12-bsb11220604-5

YINTANG, X. 2007. Flora of Xian. China (Chinese Edition). China Press. Disponible en: https://www. biodiversitylibrary.org/item/112940

ZEBALlOS, S. R., P. A. TECCO, M. CABIDO \& D. E. GURVICH. 2014. Composición de especies leñosas en comunidades invadidas en montañas del centro de Argentina: su relación con factores ambientales locales. Revista Biol. Trop. 62: 1549-1563. 